EPV208/\#485 THE CLINICAL DEMAND FOR PRESSURIZED INTRAPERITONEAL AEROSOL CHEMOTHERAPY IN SOUTH KOREA: AN ELECTRONIC SURVEY-BASED STUDY

${ }^{1}$ EJ Lee ${ }^{*},{ }^{1}$ SJ Park, ${ }^{2}$ J Mun, ${ }^{2}$ H Paik, ${ }^{2}$ J Lee, ${ }^{2}$ A Seol, ${ }^{2}$ J Kim, ${ }^{3} \mathrm{~N}$ Lee, ${ }^{4} \mathrm{GW}$ Yim, ${ }^{5} \mathrm{~S}$-H Shim, ${ }^{1} \mathrm{HS}$ Kim, ${ }^{6} \mathrm{~S}$-J Chang. ${ }^{1}$ Seoul National University College of Medicine, Obstetrics and Gynecology, Seoul, Korea, Republic of; ${ }^{2}$ Seoul National University College of Medicine, Department of Obstetrics and Gynecology, Seoul, Korea, Republic of; ${ }^{3}$ CHA Gangnam Medical Center, Obstetrics and Gynecology, Seoul, Korea, Republic of; ${ }^{4}$ Dongguk University Ilsan Hospital, Department of Obstetrics and Gynecology, Goyang, Korea, Republic of; ${ }^{5}$ Konkuk University School of Medicine, Obstetrics and Gynecology, Seoul, Korea, Republic of; ${ }^{6}$ Ajou University School of Medicine, Obstetrics and Gynecology, Suwon, Korea, Republic of

\subsection{6/ijgc-2021-IGCS.279}

Objectives Pressurized intraperitoneal aerosol chemotherapy (PIPAC) is effective for treating solid tumors with peritoneal metastasis. However, PIPAC is not a standard treatment globally and is currently only used in the limited areas. Thus, we performed a survey of surgical oncologists related to PIPAC to evaluate the clinical desire for PIPAC in South Korea, one of the many countries where PIPAC has not yet been introduced. Methods We performed an online survey of Korean surgical oncologists between November and December 2019. The questionnaire consisted of 20 questions related to PIPAC, which were divided into comprehensive inquiry (5 questions), procedure inquiry (13 questions), and cost inquiry (2 questions).

Results A total of 164 respondents answered the questionnaire. Among all respondents, 41.7-50\% majoring in ovarian cancer, pseudomyxoma peritonei, and malignant mesothelioma preferred PIPAC for the curative treatment of primary diseases, whereas $32.7-33.3 \%$ majoring in colorectal and hepatobiliary cancers chose PIPAC for the palliative treatment of recurrent diseases. Moreover, 66.7-95.2\% of the respondents considered PIPAC appropriate for the cancers the specialized in. About $70 \%$ expected a treatment response of more than 50\% through the repeated implementation of PIPAC under general anesthesia. Respondents also considered grade 1 or 2 minor surgical complications an acceptable risk. Finally, respondents considered the reasonable costs to purchase and implement PIPAC once at between 1,000,000-5,000,000 KRW.

Conclusions Although the treatment scope for applying PIPAC was different among Korean surgical oncologists, most of them expected relatively high tumor response rates with minor toxicities through the repeated implementation of PIPAC.

\section{EPV209/\#49 SURVIVAL IN CASE OF CARDIOPHRENIC LYMPHADENOPATHY IN ADVANCED STAGE EPITHELIAL OVARIAN CANCER PATIENTS WHO UNDERWENT CYTOREDUCTIVE SURGERY; A SYSTEMATIC REVIEW AND META- ANALYSIS}

\footnotetext{
${ }^{1} \mathrm{M}$ Kengsakul* ${ }^{2} \mathrm{G}$ Nieuwenhuyzen-De Boer, ${ }^{3} \mathrm{~A}$ Bijleveld, ${ }^{4} \mathrm{~S}$ Udomkarnjananun, ${ }^{5} \mathrm{~S}$ Kerr, ${ }^{6} \mathrm{C}$ Niehot, ${ }^{2} \mathrm{H}$ Van Beekhuizen. ${ }^{1}$ Panyananthaphikkhu Chonprathan Medical Center, Srinakharinwirot University, Gynecologic Oncology, Nonthaburi, Thailand; ${ }^{2}$ Erasmus MC Cancer institute, University Medical Center Rotterdam, Gynecologic Oncology, Rotterdam, Netherlands; ${ }^{3}$ Albert Schweitzer Hospital, Obstetrics and Gynecology, Dordrecht, Netherlands; ${ }^{4}$ King Chulalongkorn Memorial Hospital, Chulalongkorn University, Department of Medicine, Bangkok, Thailand; ${ }^{5}$ King Chulalongkorn Memorial Hospital, Chulalongkorn University, Research Affairs, Bangkok, Thailand; ${ }^{6}$ Erasmus MC Cancer institute, University Medical Center Rotterdam, Medical Library, Rotterdam, Netherlands
}

10.1136/ijgc-2021-IGCS.280
Objectives Favorable survival outcomes for patients with advanced stage epithelial ovarian cancer (ASEOC) is associated with complete cytoreduction. In this meta-analysis we evaluate the therapeutic role of cardiophrenic lymph nodes (CPLNs) resection ASEOC who have undergone cytoreductive surgery.

Methods Embase, Medline, Web of science, Cochrane Library and Google scholar were searched for articles published in English from their inception to November 2020. Meta-analysis was conducted to determine the prognostic impact of surgical outcome, postoperative complications and survival.

Results Fifteen relevant articles, 727 patients with CPLNs adenopathy and 981 patients without CPLNs adenopathy, were analyzed. Higher percentage of ascites, intra and extra abdominal metastases was observed in CPLNs adenopathy

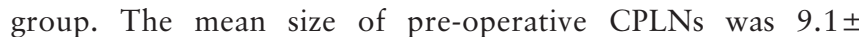
$3.75 \mathrm{~mm}$. Eighty-two percent of enlarged CPLN were histological confirmed. No difference in surgical outcome and perioperative complication was observed between both groups. Meta-analysis showed that patients with CPLNs adenopathy had a significantly increased risk of disease recurrence (OR 4.56, 95\% CI 1.98-10.51, $\mathrm{P}<0.001$ ) and dying from disease (OR 2.96, 95\% CI 2.08- 4.22, $\mathrm{p}<0.001)$ in comparison to those without CPLNs adenopathy.

Conclusions Patients with CPLNs adenopathy had higher tumor burden intra and extra-abdominally and decreased survival compared to patients without CPLNs adenopathy. There is not enough available data to confirm the therapeutic role of CPLNs resection. Therefore, a randomized controlled trial should be conducted to demonstrate the benefit of CPLNs resection in cytoreductive surgery.

\section{EPV210/\#490 PROGNOSTIC IMPLICATIONS OF HEMODYNAMIC INSTABILITY DURING OVARIAN CANCER SURGERY}

${ }^{1} \mathrm{~K} \mathrm{Kim}{ }^{*},{ }^{1} \mathrm{SI} \mathrm{Kim},{ }^{1} \mathrm{~A}$ Seol, ${ }^{1} \mathrm{HS}$ Kim, ${ }^{1} \mathrm{HH}$ Chung, ${ }^{1} \mathrm{~J}-\mathrm{W}$ Kim, ${ }^{1} \mathrm{NH}$ Park, ${ }^{1} \mathrm{Y}$-S Song, ${ }^{1,2} \mathrm{M}$ Lee. ${ }^{\prime}$ Seoul National University College of Medicine, Department of Obstetrics and Gynecology, Seoul, Korea, Republic of; ${ }^{2}$ Seoul National University Hospital, Department of Obstetrics and Gynecology, Seoul, Korea, Republic of

\subsection{6/ijgc-2021-IGCS.281}

Objectives To evaluate the impact of intraoperative hypotension and hemodynamic instability on survival outcomes in patients with high-grade serous ovarian carcinoma (HGSOC).

Methods We retrospectively identified patients with HGSOC, who underwent primary or interval debulking surgery between August 2013 and December 2019. We collected anesthesia-related variables, including the arterial blood pressure measurements (at 1-min interval) during surgery of patients. The cumulative duration of mean arterial blood pressure (MAP) readings under $65 \mathrm{mmHg}$ and two performance measurements (median performance error [MDPE] and wobble) were calculated. We investigated associations between the factors indicating hemodynamic instability and prognosis.

Results In total, 338 patients were included. Based on the cumulative duration of MAP under $65 \mathrm{mmHg}$, we divided patients into two groups: $\geq 30 \mathrm{~min}$ and $<30 \mathrm{~min}$. The progression-free survival (PFS) was worse in the $\geq 30 \mathrm{~min}$ group $(n=107)$ than the $<30$ min group $(n=231)$ (median, 18.2 vs. 